eISSN 2444-7986

DOI: https://doi.org/10.14201/orl.21022

ARTÍCULO DE REVISIÓN

\title{
TERAPIA FÍSICA EN LA HIPOFUNCIÓN VESTIBULAR UNILATERAL Y BILATERAL
}

\section{Physical therapy in unilateral and bilateral vestibular hypofunction}

José Ignacio BENITO-OREJAS ${ }^{1}$; María José AYLAGAS-ANDRÉS²; Carmen MARTÍN-MORATINOS²; Irene GALLARDO-CHAPARRO²; Laura PÉREZ-HICKMAN²; Vanesa ALADRO-ABAD ${ }^{3}$; Niria PLAZA-GARCÍA ${ }^{3}$; José María LÓPEZ-FRANCO ${ }^{3}$; Beatriz DÍEZ-RABADÁN ${ }^{3}$; Carmen GARCÍA-FRANCO ${ }^{3}$; Lucía SECO-RODRÍGUEZ; ; Felipa PANIAGUA-SERRANO ${ }^{3}$; María Elena BUSTELO-SANZ³ María Teresa JIMÉNEZ-GARCÍA ${ }^{3}$; Diana ALONSO-TOBALINA³ Serán Tamara GARCÍA-SOMOZA³ ${ }^{3}$ Ana Isabel GÓMEZ-TEJERO3; Inés ALONSO-RODRÍGUEZ³; María Jesús COLLAZOS-VILLAFÁFILA ${ }^{3}$; Irene GARCÍA-MARTÍNEZ ${ }^{3}$; Teresa BAZ-HERRERO ${ }^{3}$; Laura Alejandra MARTÍN-GUTIÉRREZ3 ${ }^{3}$ Cristina MARTÍN-TUDA ${ }^{3}$; Inés Flavia DE LA CAL-GÓMEZ³; Ana SÁNCHEZ-MARTÍNEZ ${ }^{1}$

${ }^{1}$ Médicos Servicio de ORL y PCF. ${ }^{2}$ Médicos Servicio de Rehabilitación. ${ }^{3}$ Fisioterapeutas Servicio de Rehabilitación. SACYL. Hospital Clínico Universitario de Valladolid. Valladolid. España.

Correspondencia: jbenitoorejas@gmail.com

Fecha de recepción: 4 de julio de 2019

Fecha de aceptación: 20 de julio de 2019

Fecha de publicación: 22 de julio de 2019

Fecha de publicación del fascículo: 1 de marzo de 2020

Conflicto de intereses: Los autores declaran no tener conflictos de intereses

Imágenes: Los autores declaran haber obtenido las imágenes con el permiso de los pacientes

Política de derechos y autoarchivo: se permite el autoarchivo de la versión post-print (SHERPA/RoMEO)

Licencia CC BY-NC-ND. Licencia Creative Commons Atribución-NoComercial-SinDerivar 4.0 Internacional

Universidad de Salamanca. Su comercialización está sujeta al permiso del editor

RESUMEN: Introducción: La rehabilitación vestibular (RV) basada en la terapia física, tiene el objetivo, en el caso de patología vestibular, de inducir la compensación del sistema nervioso central (SNC) a nivel de núcleos vestibulares y de otros niveles del SNC. Incluye ejercicios de habituación, adaptación y sustitución vestibular, ejercicios para mejorar el equilibrio y el control postural dinámico y ejercicios para el acondicionamiento general. En este capítulo discutimos los recientes avances sobre el adiestramiento del equilibrio y de la marcha, la estabilidad de la mirada y la habituación, en el contexto de los trastornos vestibulares uni y bilaterales. Método: Revisión narrativa. Resultados: Los ejercicios se prescriben para 
mejorar la función; fortaleciendo, y favoreciendo la flexibilidad y la resistencia, a través de la adaptación del RVO, la habituación, la sustitución sensorial, la marcha y el equilibrio postural. Son más eficaces los programas personalizados que los genéricos. El cumplimiento mejora con la personalización y las visitas de seguimiento a un fisioterapeuta. Discusión/Conclusiones: La RV permite mejorar el déficit funcional y los síntomas subjetivos derivados de la hipofunción vestibular periférica uni y bilateral, así como las alteraciones del equilibrio de origen central. Los objetivos de la RV consisten en reducir los síntomas para mejorar la estabilidad postural y de la mirada (particularmente durante los movimientos de la cabeza) y devolver al individuo a sus actividades normales, incluyendo la actividad física, la conducción y el trabajo habitual. Los médicos deben ofrecer la RV a quienes muestren limitaciones funcionales relacionadas con un déficit vestibular, pues actualmente se considera el tratamiento estándar en la disfunción vestibular periférica.

PALABRAS CLAVE: mareo; equilibrio; rehabilitación vestibular; estabilidad de la mirada; estabilidad postural; habituación.

SUMMARY: Introduction: The vestibular rehabilitation is an exercise-based method, aiming to maximize central nervous system (CNS) compensation at vestibular nuclear and other CNS levels for vestibular pathology. Vestibular rehabilitation includes exercises to habituate symptoms, exercises to promote vestibular adaptation and substitution, exercises to improve balance and dynamic postural control, and exercises to improve general conditioning. Recent advances in balance and gait training, gaze stability training, habituation training, are discussed in this chapter in the context of unilateral and bilateral vestibular disorders. Method: Narrative review. Results: Exercises are prescribed that address VOR adaptation, habituation, sensory substitution, gait and posture, strengthening, flexibility, and endurance to maximize functioning. Customized exercise programs have been shown to be more effective than providing a patient with a generic exercise program. It is thought that compliance is enhanced with customization and with follow-up visits with a physical therapist. Discussion/ conclusions: VR therapy is effective in improving functional deficits and subjective symptoms resulting from unilateral and bilateral peripheral vestibular hypo function, as well as from central balance disorders. The goals of vestibular rehabilitation are to reduce subjective symptoms, to improve gaze and postural stability (particularly during head movements), and to return the individual to normal activities, including regular physical activity, driving, and work. Clinicians should offer vestibular rehabilitation to persons with impairments and functional limitations related to the vestibular deficit. Vestibular rehabilitation is now considered the standard of care for persons with peripheral vestibular dysfunction.

KEYWORDS: dizziness; balance; vestibular rehabilitation; gaze stability; postural stability; habituation.

\section{INTRODUCCIÓN}

La mayor parte de lesiones vestibulares periféricas tienen una causa benigna y alcanzan una resolución espontánea debido a su naturaleza autolimitada o al proceso de compensación central. Ocasionalmente, incluso en ausencia de una lesión vestibular activa, se produce una compensación parcial o se utilizan unas estrategias de control postural mal adaptadas [1]. La falta de compensación ocasiona inestabilidad postural, visión borrosa con los movimientos de cabeza y sensación de mareo/desequilibrio. Es necesario un tratamiento apropiado, porque el mareo representa un riesgo importante de caídas, conllevando grandes costes directos e indirectos [2-3]. Estos pacientes son los que se beneficiarán de un programa de rehabilitación vestibular (RV), que es un tratamiento basado en un conjunto de ejercicios, elegidos para promover la adaptación y sustitución vestibular [4]. La primera terapia física o de entrenamiento del equilibrio aplicada a pacientes con alteración 


\section{TERAPIA FÍSICA EN LA HIPOFUNCIÓN VESTIBULAR UNILATERAL Y BILATERAL}

BENITO-OREJAS J I ET AL

vestibular fue propuesta en los años 40 por Sir Terence Cawthorne [5] y Harold Cooksey [6], evolucionando desde entonces con un incremento exponencial de su evidencia en los últimos 20 años, hasta convertirse actualmente, en el tratamiento estándar de los pacientes con disfunción vestibular crónica [7-9]. Exceptuando la medicación sintomática que suele requerirse en el estadio agudo y la posibilidad de un procedimiento ablativo en aquellos casos de vértigo recurrente y sintomatología incontrolada, existe suficiente evidencia, según una reciente revisión Cochrane [10], de que la RV es un tratamiento eficaz y seguro, que según se afirma en las actuales guías clínicas, debe ofrecerse a los pacientes con hipofunción vestibular uni y bilateral y a los que muestren limitaciones funcionales relacionadas con un déficit vestibular [8]. Hay 2 grandes grupos de ejercicios diferenciables, según se dirijan al tratamiento de una hipofunción vestibular (objetivo de este tema), o a la reposición de partículas en el caso del vértigo posicional paroxístico benigno (VPPB) [4], ya tratado anteriormente [11].

El sistema vestibular es una compleja red neuronal que ofrece información de los movimientos de cabeza y de la posición del cuerpo, a fin de mantener un control de la visión y del equilibrio. Está organizado en 2 unidades funcionales diferentes: el sistema vestíbulo-ocular responsable de la estabilidad visual durante los movimientos de la cabeza y el sistema vestíbulo espinal, encargado del control postural [12]. De acuerdo a la neurofisiología, la afectación vestíbulo-ocular se manifiesta por mareo/vértigo e inestabilidad visual, mientras que la disfunción vestíbulo-espinal provoca desequilibrio. Dado que ambas funciones no comparten idénticos circuitos neuronales, es posible que el grado de afectación y la sintomatología observada en el tiempo sean diferentes. Por tal motivo, los actuales programas de RV pretenden ser de carácter individual, adaptados a las características de la lesión, y acordes a las expectativas del individuo; prolongándose en el tiempo lo necesario hasta conseguir la curación o la estabilización clínica; de dificultad progresiva y adecuada a la situación funcional del paciente y, a ser posible, integrándose en las actividades de la vida cotidiana. La edad no parece afectar los resultados de esta terapia [13].

En términos generales constan de 4 diferentes tipos de ejercicios, utilizados en función de las limitaciones que se hubieran identificado durante la evaluación $[4,7-9,14]$ : 1. Ejercicios para mejorar la estabilidad de la mirada. 2. Ejercicios de habituación. 3. Ejercicios para recuperar el equilibrio y la marcha. 4. Ejercicio físico de acondicionamiento general.

\section{EJERCICIOS DE ESTABILIDAD DE LA MIRADA (TABLAS 1 Y 2, FIGURA 1)}

La capacidad de mantener enfocado un objeto cuando la cabeza se mueve (estabilidad dinámica de la mirada) es mediada por el RVO, el reflejo cérvico-ocular (RCO) y el sistema visual a través de las vías correspondientes al seguimiento lento $\mathrm{y}$ al optoquinético $(\mathrm{OKN})$ [15]. Con alteraciones vestibulares tanto de origen periférico como central, la ganancia del reflejo vestíbulo ocular (RVO) puede incrementarse o disminuir, ocasionando visión borrosa [7]. Las velocidades del ojo y de la cabeza deben ser muy precisas para mantener la fijación de la mirada durante los movimientos de la cabeza. La nitidez de una imagen visual cuando la cabeza se mueve a la derecha se obtiene si los ojos se desplazan hacia la izquierda de manera simultánea y en la misma medida (velocidad del ojo/velocidad de la cabeza $=1$ ) [7].

En la actualidad, los ejercicios de estabilidad de la mirada que se prescriben a quienes tienen alteraciones vestibulares, buscan inducir el deslizamiento retiniano [16], por ser uno de los mecanismos a través del que se consigue disminuir la visión borrosa durante los movimientos de la cabeza [17]. Este deslizamiento retiniano puede provocarse con movimientos de cabeza horizontales o verticales, mientras se mantiene la fijación visual en una diana, 


\section{TERAPIA FÍSICA EN LA HIPOFUNCIÓN VESTIBULAR UNILATERAL Y BILATERAL BENITO-OREJAS J I ET AL}

fija o en movimiento. La diana puede sujetarse con el brazo estirado o colocarse en cualquier lugar de una habitación (Tabla 1 y Figura 1) [18]. Estudios experimentales en primates y humanos han demostrado que, la repetición de estos ejercicios induce la adaptación del RVO, [19-20], siendo más efectivos los movimientos activos que los pasivos [14]. Para incrementar la magnificación y duración de este deslizamiento, el paciente puede fijarse en una diana que se mueve en dirección opuesta a la cabeza (RVOX2) (Tabla 1 y Figura 1) [21]. Es importante realizar los ejercicios de estabilidad de la mirada con buena visión y por tanto con la mejor luz posible [22].

Pero también hay ejercicios de estabilidad de la mirada, basados en el principio de sustitución, desarrollados con la finalidad de promover estrategias alternativas (como movimientos de seguimiento lento o facilitando los mecanismos centrales de pre-programación del movimiento ocular) para sustituir una falta completa de función vestibular [23]. Así, por ejemplo, los ejercicios entre dianas consisten en desviar la mirada a una diana antes de desplazar la cabeza hacia la misma, estimulando potencialmente el uso de movimientos preprogramados de los ojos [8,24] (Tabla 1 y Figura 1).

Tanto los ejercicios de adaptación como los de sustitución se realizarán en sentido horizontal (diciendo «no» con la cabeza) y vertical («si»), no siendo tan efectivos los movimientos laterales («quizás») [21]. También se variará la frecuencia de los movimientos, pues los mayores cambios del

Tabla 1. Ejercicios para mejorar la estabilidad de la mirada

RVO X1:

Una tarjeta de visita con una palabra (visión foveal), se coloca enfrente del paciente, en la pared, de forma que pueda leerla. El paciente mueve su cabeza durante 1 minuto de un lado hacia otro, manteniendo la visión de la palabra. Utilizar diferentes velocidades y añadir un fondo desestabilizante (tablero de ajedrez, persiana entreabierta...).

Se repite otro minuto, pero con movimientos verticales.

Los ejercicios se realizan, al menos 3 , pero mejor 4 o 5 veces al día.

La duración de cada ejercicio avanza de 1 a 2 minutos.

Al paciente se le avisa de que los ejercicios le pueden marear e incluso generar alguna nausea, pero que debe persistir en mantenerlos de forma continua 1 a 2 minutos, dejando 1 minuto de descanso entre cada ejercicio.

RVO X2:

Manteniendo una tarjeta en su mano con una palabra visible, mueva la cabeza y la mano en direcciones horizontales opuestas, sin perder la palabra, durante 1 minuto sin parar. Se realiza también con movimientos verticales y con un fondo desestabilizador, aumentando el tiempo de 1 a 2 minutos. El paciente repite estos ejercicios al menos 3 y preferiblemente 5 veces al día. EJERCICIOS DE OJO/CABEZA:

Podemos añadir a los anteriores, estos ejercicios. Al principio con solo 2 dianas y con movimientos horizontales. El ejercicio se realiza durante 2 minutos, pero el paciente pude descansar cuando quiera. La progresión es con movimientos verticales o con más dianas. Lo puede también hacer extendiendo sus 2 pulgares a modo de dianas.

- Sacadas: 2 dianas cercanas. Se mira de una a otra, sin mover la cabeza.

- Seguimiento y cancelación del RVO: cabeza al frente, se extiende un brazo y se mira al pulgar que se mueve lentamente de uno a otro lado.

- Sacadas y RVO: 2 dianas (o ambos brazos separados y ambos pulgares). La cabeza se dirige y mira uno, luego se mira al otro y entonces se gira la cabeza.

- Seguimiento imaginario: se mira una diana con ojos abiertos, se cierran los ojos, se mueve lentamente la cabeza, imaginando la posición de la diana y se abren los ojos, ajustando la posición. Se realiza varias veces en ambas direcciones. El ejercicio de recordar una diana se puede añadir a los ejercicios domiciliarios. 2 minutos, descansando cuando quiera y se puede complicar utilizando un láser en la cabeza.

OTROS EJERCICIOS:

- Sentado, con la cabeza inmóvil sujeta en ambas manos y la vista al frente lee un libro, sólo moviendo los ojos. Puede ir reduciendo el tamaño de las letras. Repite lo anterior, girando la cabeza de un lado hacia otro.

- Mirando una imagen (libro, TV, su dedo pulgar...) sentado en un taburete giratorio, mover el cuerpo de uno a otro lado. 
RVO ocurren en la frecuencia de entrenamiento [25,26], que se irá modificando poco a poco, ya que el incremento progresivo de la señal de error es más efectivo que la realización de movimientos bruscos, que generan grandes deslizamientos retinianos [27]. Hay también otros ejercicios que promueven este deslizamiento: como el movimiento de la cabeza con ojos cerrados intentando mantener mentalmente la posición de una diana o la búsqueda de dianas con una luz en la cabeza [27], e incluso se puede mejorar el RVO en ausencia de estímulo visual, imaginando en oscuridad una diana mientras se mueve la cabeza. También se recupera la estabilidad, ejercitando la supresión del RVO, fijando la mirada en el pulgar del propio brazo extendido mientras se desplaza en bloque la cabeza y el tronco en un sillón rotatorio [28] (Tabla 1 y Figura 1).

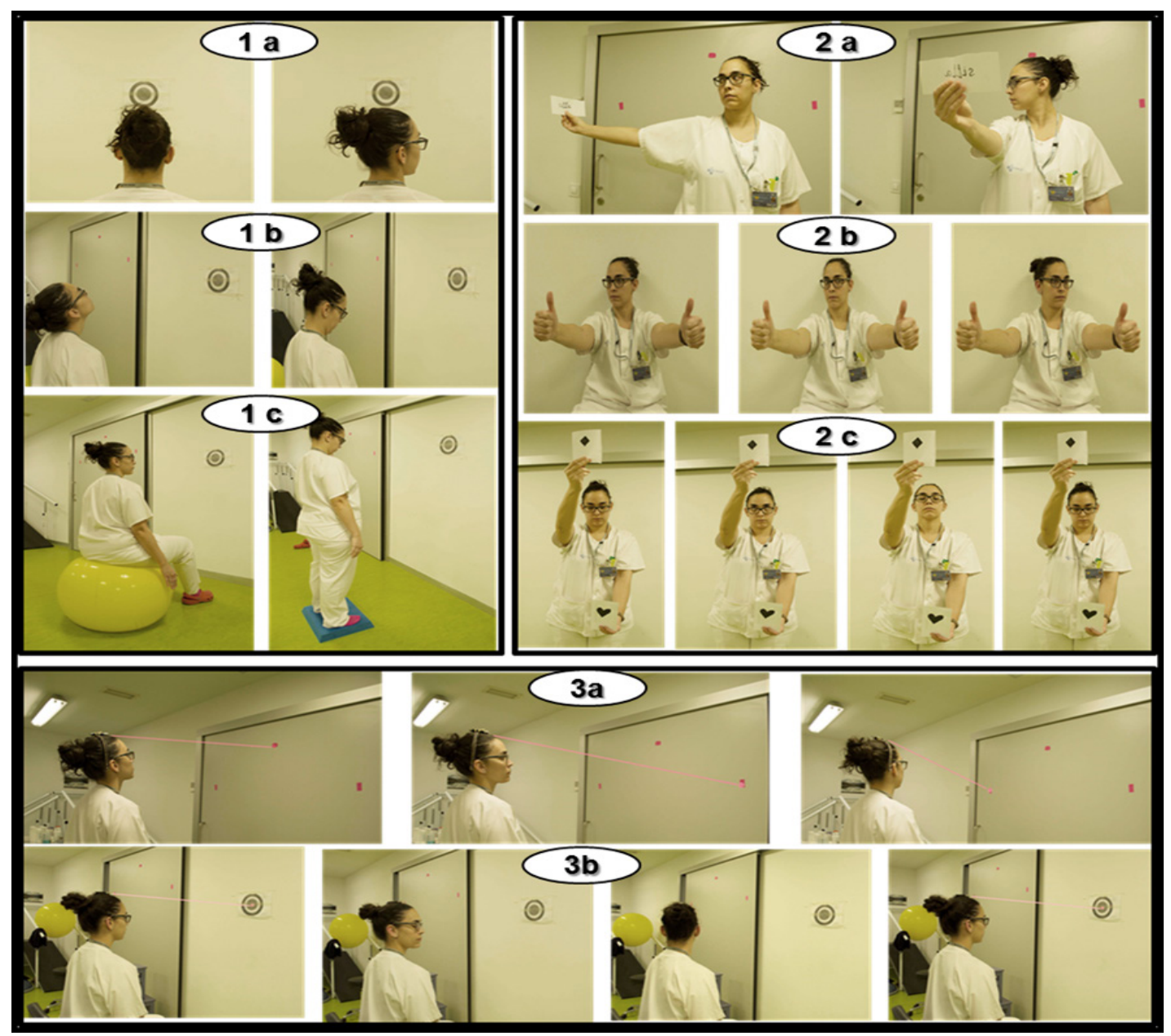

Figura 1. Ejercicios de estabilidad de la mirada. 1: ejercicios X1, en dirección horizontal (1a), vertical (1b) y sobre diferentes superficies (1c). 2a: ejercicios X2. Ejercicios de ojo/cabeza: 2b: sacada y RVO entre 2 pulgares en horizontal y $2 c$ en vertical. 3a: con un láser en la frente ejercicio de sacada y RVO entre 3 dianas. 3b: seguimiento imaginario. 


\section{TERAPIA FÍSICA EN LA HIPOFUNCIÓN VESTIBULAR UNILATERAL Y BILATERAL BENITO-OREJAS J I ET AL}

Generalmente los ejercicios irán progresando: sentado, de pie, caminando; alterando el fondo, haciéndolo visualmente más complejo (tablero de ajedrez, mantel de cuadros, luz entrando por una persiana entreabierta, etc.) y modificando la superficie de soporte (lisa y dura, alfombra, gomaespuma e incluso arena) [14] (Tabla 2). Los pacientes realizarán estos ejercicios de 3 a 5 veces al día, durante 12-20 minutos/día, además de unos 20 minutos de adiestramiento del equilibrio y la marcha [29].

Tabla 2. Progresión de los ejercicios de estabilidad de la mirada (Adaptado de Herdmann SJ y Clendaniel RA [41])

\begin{tabular}{|c|c|c|c|}
\hline Ejercicio & Duración & Frecuencia & Posición \\
\hline $\begin{array}{l}\mathrm{X} 1 \text { en pared, con movimientos de cabeza } \\
\text { horizontales y verticales. }\end{array}$ & $\begin{array}{l}\text { Menos de } 1 \text { minuto } \\
\text { cada vez }\end{array}$ & 2 o 3 veces al día & $\begin{array}{l}\text { Primero sentado y cuando se haga } \\
\text { bien, de pie. }\end{array}$ \\
\hline $\begin{array}{l}\mathrm{X} 1 \text { en pared, con movimientos de cabeza } \\
\text { horizontales y verticales. }\end{array}$ & $\begin{array}{l}\text { Incrementar a } \\
1 \text { minuto cada } \\
\text { ejercicio }\end{array}$ & 5 veces al día & $\begin{array}{l}\text { De pie (e incluso se pueden hacer } \\
\text { más complejos, cambiando la base } \\
\text { de soporte). }\end{array}$ \\
\hline $\begin{array}{l}\text { X1 sujetando la diana con la mano, con } \\
\text { movimientos de cabeza horizontales y } \\
\text { verticales. }\end{array}$ & $\begin{array}{l}1 \text { minuto cada } \\
\text { ejercicio }\end{array}$ & Más de 5 veces al día & $\begin{array}{l}\text { De pie (y/o cambiando la base de } \\
\text { soporte). }\end{array}$ \\
\hline $\begin{array}{l}\text { Añade ejercicios de ojo/cabeza horizontal } \\
\text { y vertical }\end{array}$ & Un rato & 2 o 3 veces al día & Primero sentado y después de pie. \\
\hline $\begin{array}{l}\text { X1 con diana en la mano y otra más } \\
\text { alejada en la pared }\end{array}$ & $\begin{array}{l}1 \text { minuto cada } \\
\text { ejercicio }\end{array}$ & 2 o 3 veces al día & $\begin{array}{l}\text { De pie (y/o cambiando la base de } \\
\text { soporte). }\end{array}$ \\
\hline $\begin{array}{l}\text { Añade ejercicios de ojo/cabeza horizontal } \\
\text { y vertical }\end{array}$ & Un rato & 2 o 3 veces al día & $\begin{array}{l}\text { De pie (y/o cambiando la base de } \\
\text { soporte). }\end{array}$ \\
\hline $\begin{array}{l}\text { X1 con diana en la mano y otra más } \\
\text { alejada en la pared }\end{array}$ & $\begin{array}{l}1 \text { minuto cada } \\
\text { ejercicio }\end{array}$ & 4 veces al día & $\begin{array}{l}\text { De pie (y/o cambiando la base de } \\
\text { soporte). }\end{array}$ \\
\hline $\begin{array}{l}\text { Añade ejercicios de ojo/cabeza horizontal } \\
\text { y vertical }\end{array}$ & Un rato & 4 veces al día & $\begin{array}{l}\text { De pie (y/o cambiando la base de } \\
\text { soporte). }\end{array}$ \\
\hline Añade ejercicios de diana imaginaria & Un rato & 4 veces al día & Primero sentado y después de pie. \\
\hline $\begin{array}{l}\text { X1 con diana en la mano y otra más } \\
\text { alejada en la pared }\end{array}$ & $\begin{array}{l}1 \text { minuto cada } \\
\text { ejercicio }\end{array}$ & 4 veces al día & $\begin{array}{l}\text { De pie (y/o cambiando la base de } \\
\text { soporte). }\end{array}$ \\
\hline $\begin{array}{l}\text { Añade ejercicios de ojo/cabeza horizontal } \\
\text { y vertical }\end{array}$ & Un rato & 4 veces al día & $\begin{array}{l}\text { De pie (y/o cambiando la base de } \\
\text { soporte). }\end{array}$ \\
\hline Añade ejercicios de diana imaginaria & Un rato & 4 veces al día & $\begin{array}{l}\text { De pie (y/o cambiando la base de } \\
\text { soporte). }\end{array}$ \\
\hline $\begin{array}{l}\text { Algunos pacientes pueden progresar a X2 } \\
\text { con diana en la mano y a distancia }\end{array}$ & $\begin{array}{l}1 \text { minuto cada } \\
\text { ejercicio }\end{array}$ & 4 veces al día & $\begin{array}{l}\text { De pie (y/o cambiando la base de } \\
\text { soporte). }\end{array}$ \\
\hline $\begin{array}{l}\text { Añade ejercicios de ojo/cabeza horizontal } \\
\text { y vertical }\end{array}$ & Un rato & 4 veces al día & $\begin{array}{l}\text { De pie (y/o cambiando la base de } \\
\text { soporte). }\end{array}$ \\
\hline Añade ejercicios de diana imaginaria & Un rato & 4 veces al día & $\begin{array}{l}\text { De pie (y/o cambiando la base de } \\
\text { soporte). }\end{array}$ \\
\hline $\mathrm{X} 1$ con diana en la mano y a distancia & $\begin{array}{l}1 \text { minuto cada } \\
\text { ejercicio }\end{array}$ & 4 veces al día & $\begin{array}{l}\text { De pie (y/o cambiando la base de } \\
\text { soporte). }\end{array}$ \\
\hline $\begin{array}{l}\text { Añade ejercicios de ojo/cabeza horizontal } \\
\text { y vertical }\end{array}$ & Un rato & 4 veces al día & $\begin{array}{l}\text { De pie (y/o cambiando la base de } \\
\text { soporte). }\end{array}$ \\
\hline Añade ejercicios de diana imaginaria & Un rato & 4 veces al día & $\begin{array}{l}\text { De pie (y/o cambiando la base de } \\
\text { soporte). }\end{array}$ \\
\hline $\begin{array}{l}\text { Ir buscando números, escritos de forma } \\
\text { aleatoria en una pared }\end{array}$ & Un rato & 2 veces al día & $\begin{array}{l}\text { De pie y dando un paso para señalar } \\
\text { el número encontrado. }\end{array}$ \\
\hline
\end{tabular}




\section{TERAPIA FÍSICA EN LA HIPOFUNCIÓN VESTIBULAR UNILATERAL Y BILATERAL BENITO-OREJAS J I ET AL}

Pero los ejercicios de estabilidad de la mirada no sólo acrecientan la agudeza visual a través de la adaptación del RVO, sino también a consecuencia del incremento en el número de sacadas compensatorias [14]. En pacientes con hipofunción vestibular uni o bilateral, se reduce el error en la posición de la mirada si se generan estas sacadas, como estrategia para estabilizar la mirada [24], lo que mejora la visión borrosa y el mareo [30]. Antes de los trabajos de Schubert et al., 2010 [24, 27] se pensaba que la recuperación de los síntomas dependía exclusivamente de los cambios de ganancia del RVO, pero parece que hay una combinación de este mecanismo con el de sustitución sacádica que, en conjunto, contribuyen a la reducción de la visión borrosa durante los movimientos de la cabeza. El uso de métodos computarizados para registrar la prueba impulsiva de la cabeza, son los que han permitido un mayor conocimiento de los mecanismos que hacen posible la adaptación del RVO [24,31].

Otra forma de inducir el deslizamiento retiniano es mediante el estímulo optoquinético $(\mathrm{OKN})$, que no requiere movimientos de cabeza. La dirección del estímulo OKN incrementa la respuesta vestibular en la misma dirección, y puede usarse para mejorar la ganancia del RVO en disfunciones periféricas unilaterales [32].

\section{EJERCICIOS DE HABITUACIÓN}

La habituación es otro concepto utilizado en la rehabilitación de pacientes con mareo/desequilibrio, basado en el hecho de que la repetición de una maniobra que provoca síntomas disminuye los mismos [14]. Los ejercicios de habituación se eligen en función de aquellos movimientos o situaciones que producen mareo/vértigo, debiendo diferenciar un VPPB (que requiere maniobras propias) de un vértigo de posición, consecuencia de una pobre compensación tras un daño laberíntico, donde usaremos la habituación [1]. La mejoría se suele conseguir en un periodo de 4 a 6 semanas.
Actualmente se ha incorporado el estímulo OKN y la realidad virtual en los ejercicios de habituación, siendo utilizados en casos de sensibilidad al movimiento visual (que analizaremos en otro capítulo) [8].

\section{EJERCICIOS DE EQUILIBRIO Y DEMARCHA} (TABLA 3 Y FIGURAS 2 Y 3)

La recuperación de la estabilidad postural es más lenta que la de la mirada [33]. Los ejercicios pretenden facilitar el uso de las entradas visual y somatosensorial, para sustituir la función vestibular deficiente. Para ello, forzamos el equilibrio del paciente alterando la entrada visual (ojos cerrados, estímulo OKN, realidad virtual) y/o la somatosensorial (gomaespuma o superficies en movimiento), con modificaciones de la base de soporte (Romberg, tándem, de pie sobre una pierna), a fin de incrementar el conflicto sensorial (Tabla 3 y Figura 2). En resumen, se trata de enseñar al paciente a utilizar referencias visuales estables y servirse de la información somatosensorial, como los principales sistemas para su control postural, con independencia de que también se pretenda estimular la función vestibular residual [4]. En general, los pacientes confían en la información somatosensorial procedente de sus extremidades inferiores en la fase aguda de una hipofunción vestibular unilateral y en la entrada visual durante el estadio crónico [21], pero en ocasiones se produce una dependencia visual o somatosensorial, que resulta perjudicial [4] y de la que hablaremos en otro capítulo.

El balanceo del cuerpo estando de pie, sirve para mejorar el control del centro de gravedad, el equilibrio y la recuperación de las estrategias posturales normales [8]. Hay 3 estrategias posturales que se utilizan para recuperar el equilibrio estando de pie: la de tobillo, la de cadera y la ejecución del paso cuando se superan los límites de estabilidad. La estrategia de cadera es más dependiente de la función vestibular [34], de forma que los pacientes 


\title{
TERAPIA FÍSICA EN LA HIPOFUNCIÓN VESTIBULAR UNILATERAL Y BILATERAL BENITO-OREJAS J I ET AL
}

con déficit vestibular utilizan la estrategia de tobillo en vez de la de cadera incluso cuando ésta es requerida para mantener la estabilidad (mantenerse sobre un pie, caminar por una superficie estrecha o hacer punta/talón), lo que puede facilitar la caída [35]. Al paciente se le entrena en una determinada

Tabla 3. Ejercicios para mejorar la estabilidad postural (Modificado de Herdmann SJ y Whitney SL [33]).

\begin{abstract}
Equilibrio estático:
Pueden utilizarse múltiples ejercicios de equilibrio. Estos tienen la finalidad de introducir los movimientos de cabeza y forzar el uso de diferentes apoyos para mantener el equilibrio.

1. El paciente está de pie, sobre una superficie firme, con los pies separados a la altura de los hombros, mirando de frente a una diana en la pared. Progresivamente estrecha su base de sustentación: de pies separados a pies juntos, medio pie delante y el otro medio detrás e incluso en tándem (un pie delante del otro). El ejercicio se realiza primero con los brazos separados y luego pegados al cuerpo y finalmente cruzados en el pecho. Cada posición se mantiene 15-30s, antes de intentar la siguiente más difícil. Se practica durante 2 a 10 minutos. También se puede intentar hacer con los ojos cerrados. primero se cierran intermitentemente y luego por períodos cada vez más largos.

2. El paciente de pie con los pies tan juntos como pueda. Mirando una diana de frente, gira su cabeza a derecha e izda., sin perder la diana, durante 1 minuto. El ejercicio puede iniciarse apoyando inicialmente una mano en la pared, si es necesario. Intenta entonces soltar la mano durante periodos cada vez más largos. La progresión se consigue juntando todo lo posible los pies.
\end{abstract}

3. Se coloca una lámpara o un puntero, en la cintura, hombros o cabeza del paciente y éste desplaza su cuerpo para colocar la luz en diferentes dianas de la pared. Se puede realizar con los pies en diferentes posiciones y sobre superficies de diferente densidad.

4. El paciente practica sobre superficies de diferentes densidades. Los ejercicios se realizan con ojos cerrados, pero el paciente abre los ojos cuando mueve sus pies. Caminar sobre un colchón: ojos abiertos, cerrados, moviendo la cabeza (como si dijera con la cabeza: "si", "no", "tal vez")

5. Sobre una pierna intentando mantener el equilibrio. La pierna que se eleva se puede apoyar suavemente sobre un vaso de plástico invertido.

6. Sobre un balancín o plataforma móvil, se le pide al paciente que encuentre el equilibrio, cabeza inmóvil, ojos abiertos y después cerrados

7. Sobre superficie firme o incluso de gomaespuma si puede, se combina un balón con un compañero. Equilibrio dinámico, caminando:

1. El paciente camina, acompañado por alguien si es necesario.

2. El paciente camina solo y comienza a girar la cabeza hacia los lados. dado que le puede desestabilizar lo hace cerca de una pared.

3. El acompañante realiza una presión continua y lateral sobre un hombro del paciente, según camina y luego sobre el contrario, pidiéndole que mantenga la estabilidad.

4. Al paciente caminando, se le pide que realice ejercicios mentales (contar hacia atrás), moviendo la cabeza y sobre superficies de diferente densidad.

5. El paciente intenta caminar sobre una estrecha base de soporte. inicialmente sujetándose a la pared, después de forma intermitente y finalmente sin sujetarse.

6. El paciente gira $180^{\circ}$ según camina (cambio de sentido), al principio en círculos grandes y posteriormente más cerrados. Se debe girar en ambas direcciones. Se puede intentar en algún paciente incluso con ojos cerrados.

7. Se puede practicar el caminar subiendo o bajando rampas, con diferentes superficies.

8. Puede jugar con una pelota de tenis dando patadas contra una pared.

9. Se le entrenará a jugar con un balón (mejor de colores brillantes), que el paciente mirará fijamente, llevándolo desde encima de la cabeza hasta el suelo, para pasarlo entre las piernas y tirárselo al terapeuta/acompañante. Tirarse la pelota, de uno a otro, rápido y moviéndose.

10. Puede practicar en el exterior pasear por una alameda o por un supermercado (inicialmente a favor de la gente y luego en contra).

11. Puede mantener el equilibrio sentado sobre una pelota suiza o balanceándose en un trampolín. Estos ejercicios se pueden realizar fijándose en una diana, a fin de facilitar la adaptación de los reflejos otolítico-oculares.

12. Caminar y hacer cálculos mentales se puede intentar, pero es muy difícil en pacientes con déficit vestibular bilateral. 


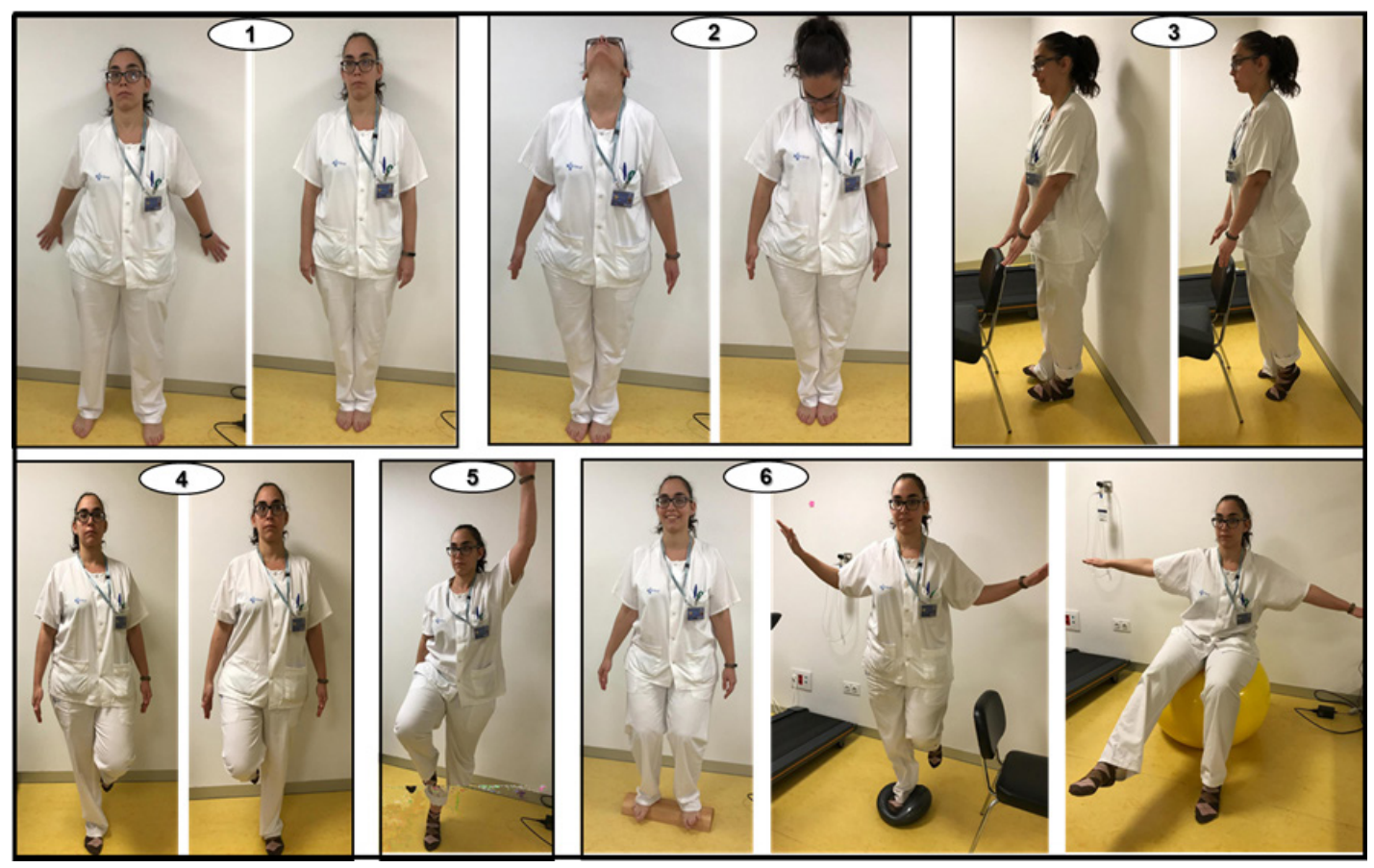

Figura 2. Ejercicios para mejorar el equilibrio postural estático. 1: sobre superficie firme y cerca de la pared (para sujetarse si se precisa), se va disminuyendo la base de sustentación; 2: con los pies juntos se mueve la cabeza en diferentes direcciones (fijándose o no en una diana); 3: movimientos de balanceo de tobillo (de talón y de puntas); 4: equilibrio sobre una y otra pierna; 5: lo mismo, levantando el brazo contrario y 6: sobre diferentes superficies.

estrategia mediante su propio balanceo o realizando tareas concretas o en respuesta a determinadas perturbaciones [4] (Figura 2).

La dificultad para caminar conduce a un declive funcional, pérdida de independencia y caídas; por lo que entrenar la marcha resulta fundamental [29]. El entrenamiento comienza en superficies lisas y posteriormente irregulares, donde se pueden añadir multitud de tareas: giros de cabeza, caminar sobre o alrededor de un obstáculo, a variable velocidad, parando y reiniciando, caminando hacia atrás o con ojos cerrados, o realizando una tarea secundaria mientras se camina (contar hacia atrás, contestar a preguntas...) [36] (Tabla 3 y Figura 3). Ejercitar la parte de la marcha más afectada, permite obtener un patrón más seguro y eficiente. Es posible añadir aquella tecnología de que se disponga, como juegos de equilibrio, estímulo OKN, sistemas de realidad virtual, feedback vibrotáctil [37-38] y la práctica del Tai-Chi que parece mejorar la sustitución sensorial incrementando la sensibilidad distal [39]. También se ha descrito la eficacia de una fisioterapia acuática [40]. En todo caso, se debe insistir en la importancia de la seguridad, especialmente en los programas de rehabilitación domiciliaria [37-38].

Reflejos cervicales: Los pacientes con déficit vestibular disminuyen los giros de cabeza para evitar el mareo y típicamente "se mueven en bloque", lo que puede conducir a una afectación musculoesquelética secundaria, con dolor de cuello y espalda [41]. Esta estrategia es de poca utilidad porque genera una limitación de la actividad normal [21]. 


\section{TERAPIA FÍSICA EN LA HIPOFUNCIÓN VESTIBULAR UNILATERAL Y BILATERAL BENITO-OREJAS J I ET AL}

Aunque el mareo cervical es considerado un diagnóstico de exclusión e históricamente cuestionado [42], con independencia del debate académico, es importante tener en cuenta la disfunción cervical, pues para realizar una correcta $\mathrm{RV}$, se deben tolerar los movimientos de cuello [43]. El reflejo cérvicoocular (RCO) provoca un movimiento rotatorio de los ojos en dirección opuesta al giro de la cabeza en frecuencias inferiores a $0,5 \mathrm{~Hz}$ y por tanto de insignificante contribución al RVO en personas normales [44]. Sin embargo, en déficits vestibulares bilaterales el RCO gana importancia, porque contribuye a generar la sacada anticompensatoria, que lleva a los ojos en dirección a la diana [44].

\section{CONDICIÓN FÍSICA GENERAL}

Caminar o realizar ejercicio aérobico es importante, porque a menudo se limita la actividad física para evitar la provocación de los síntomas [8]. Su desarrollo, mantiene la compensación adquirida [4]. Se suelen sugerir ejercicios de acuerdo con la edad, al estado de salud y al interés particular. A la mayoría, se les prescribe un programa progresivo de hasta 30 minutos de paseo diario, pero en función de las capacidades se puede realizar cualquier actividad. Los deportes que precisan coordinación de la cabeza, de los ojos y el cuerpo, como los de pala o pelota..., son muy apropiados (Tabla 3 y Figura 3). La natación debe realizarse con precaución pues es frecuente la desorientación de estos pacientes en el medio acuático [1]. En personas mayores el hablar mientras se camina, facilita la caída y por tanto se les debe instruir a que se detengan cuando quieran hablar [45]. Si los movimientos rápidos de cabeza generan mareo/desequilibrio, el paciente no debe conducir [46].

En definitiva, el objetivo final de la RV es integrar al paciente en la actividad normal de su vida diaria, incorporándose al trabajo y sintiéndose recuperado [4].

Ediciones Universidad de Salamanca / @@@

\section{FRECUENCIA Y DURACIÓN DE LOS EJERCICIOS}

Dada la heterogeneidad de las publicaciones que utilizan la RV, no hay ninguna evidencia respecto a la dosis (frecuencia, intensidad, tiempo utilizado), pudiendo incluso ser útil una simple demostración de los ejercicios, que el paciente realizará posteriormente en casa. Sin embargo, basándose en opiniones de expertos extrapoladas de la evidencia, los ejercicios de estabilidad de la mirada se realizarán por lo menos 3 veces al día y durante un mínimo de 12 minutos diarios en los pacientes con déficit vestibular agudo/subagudo y hasta 20 minutos, si la afectación vestibular es crónica [8]. Aunque el número medio de visitas supervisadas referidas en la literatura es de alrededor de 10, podríamos considerar como norma que, en los pacientes sin comorbilidades importantes que afecten a la movilidad y con una hipofunción vestibular aguda/subaguda, sería suficiente un control semanal, durante 2-3 semanas; si la hipofunción vestibular es crónica, las revisiones se prolongarán a 4-6 semanas y si la afectación es bilateral será necesario continuar hasta las 8-12 semanas. Además de estas visitas supervisadas, a los pacientes se les dará un programa de ejercicios domiciliarios diarios [8].

\section{HIPOFUNCIÓN VESTIBULAR UNILATERAL}

En la neuritis vestibular, una terapia física habitual, consistente en movimientos horizontales y verticales de cabeza, junto a un entrenamiento del equilibrio y de la marcha, mejoran la sintomatología del paciente, sustituyendo incluso al tratamiento con corticoides [47] (Tablas 1-3 y Figuras 1-3). Esta terapia física facilita la compensación central [48-49].

La terapia rehabilitadora en la enfermedad de Ménière siempre ha sido compleja dados los episodios de vértigo recurrente, utilizándose los ejercicios vestibulares para recuperar la calidad de vida [50]. Hoy en día se está aplicando la realidad virtual como programa de RV, para mejorar no sólo esta 


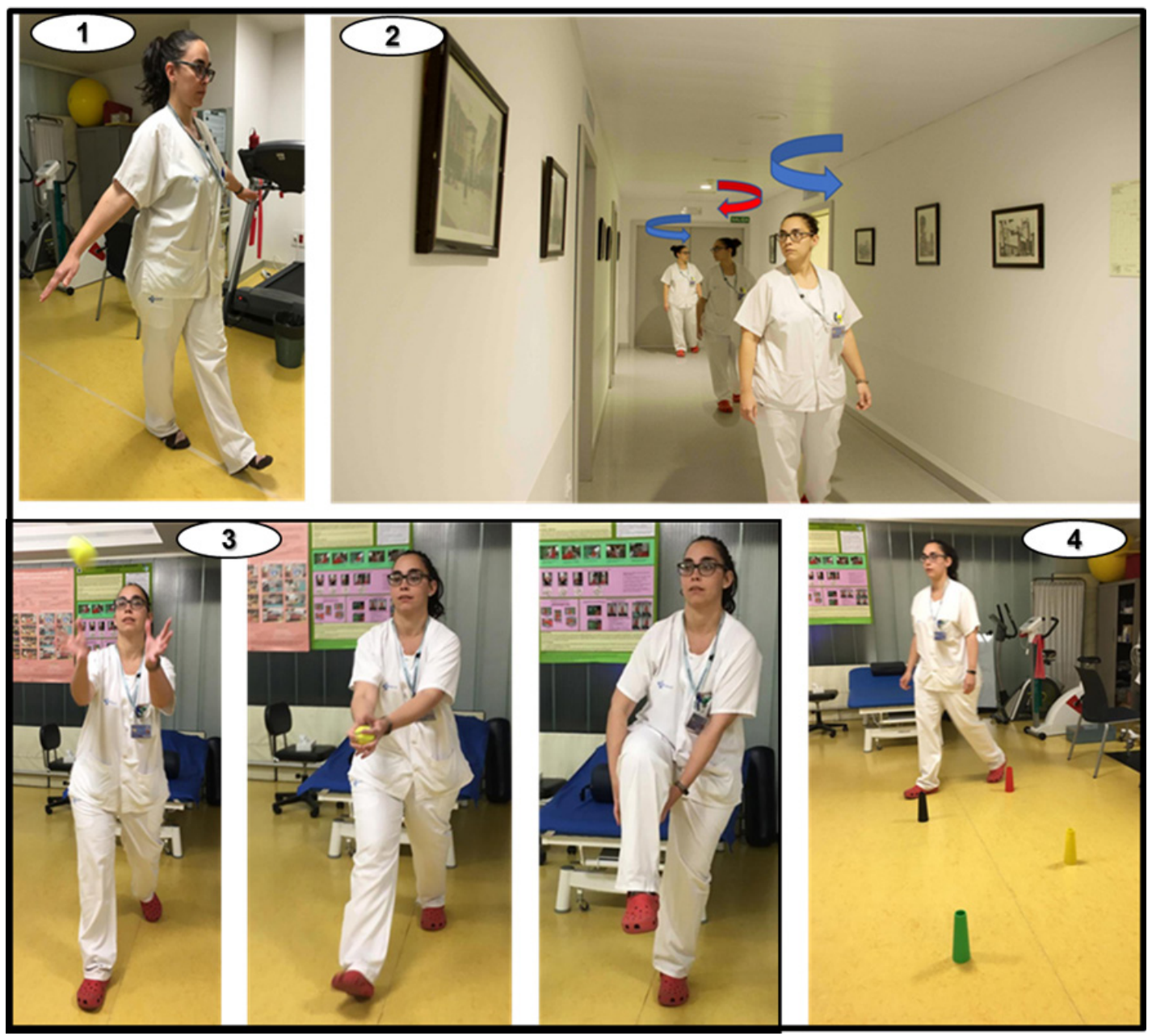

Figura 3. Ejercicios para mejorar el equilibrio postural dinámico. 1: el paciente camina, con los pies cada vez más juntos hasta conseguir una marcha en tándem; 2: camina girando la cabeza hacia ambos lados, fijando la vista en un objeto; 3: practica diferentes juegos de pelota; 4: realiza circuitos (giros, rampas, marcha atrás, con detenciones, con obstáculos...) en función de las dificultades que presente.

calidad de vida, sino también la frecuencia de las crisis y la estabilidad postural. Además, parece que no se necesitan equipos costosos, pues este restablecimiento es también posible mediante la aplicación de procedimientos económicos $[51,52,53]$.

En pacientes con alteraciones vestibulares de carácter crónico, la estabilidad visual es uno de los síntomas que más afectan al equilibrio [54]. Pavlou et al, 2013 [55] realizaron un estudio mediante sesiones de entrenamiento con estímulo optoquinético $(\mathrm{OKN})$ y tanto las visitas supervisadas como las no supervisadas mejoraron la estabilidad postural, el vértigo visual y el estado psicológico del paciente. 


\section{TERAPIA FÍSICA EN LA HIPOFUNCIÓN VESTIBULAR UNILATERAL Y BILATERAL BENITO-OREJAS J I ET AL}

HiPOFUNCIÓN VESTIBULAR BILATERAL (sÍNDROME DE DANDY)

Genera oscilopsia, mayor riesgo de caídas e importantes limitaciones. Aunque es poco frecuente, las consecuencias funcionales de una pérdida bilateral completa pueden hacer muy difícil la vida de un individuo. Dada la ausencia de RVO, se considera importante el entrenamiento sacádico entre dianas colocadas horizontal o verticalmente y realizando, como ya se ha expuesto, una progresión de sentado a de pie, caminando y sobre diversas superficies [14]. Una revisión sistemática reciente sugiere que hay poca evidencia de que la terapia física mejore el equilibrio y la estabilidad de la mirada, en estos casos [56]. Dada la mayor posibilidad de caída, aconsejaremos el uso de algún medio auxiliar de apoyo (bastón o andador), sobre todo en mayores de 65 años [2], siempre atentos a que el paciente no genere una dependencia a estas ayudas [4].

Dadas estas limitaciones funcionales, investigadores de todo el mundo trabajan por descubrir una prótesis similar al implante coclear [57] que recupere el equilibrio y la estabilidad de la mirada. Otra posibilidad tecnológica prometedora consiste en la aplicación de un feedback auditivo y vibrotáctil, donde ciertos sonidos o vibraciones ofrecen una entrada externa que informa del balanceo postural, ayudando a evitar la pérdida del equilibrio $[37,38,58,59]$.

\section{Patología vestibular central}

Hay cada vez más evidencia de que los trastornos vestibulares centrales mejoran con RV, pudiendo beneficiarse la contusión cerebral, el ictus y la migraña vestibular.

Los que sufren un traumatismo cerebral leve, con mareos, se recuperan con la RV [60]. Alsalaheen et al, 2013 [61] publicaron el tipo de ejercicios que normalmente prescriben los rehabilitadores en estos casos. Los ictus localizados en el tronco cerebral consecuencia de alteraciones en la circulación posterior, ocasionan con frecuencia alteraciones vestibulares centrales que se manifiesta por desequilibrio y vértigo, donde la terapia física también parece ser efectiva [62]. Finalmente, la migraña es un desorden neurológico muy frecuente que puede asociarse con síntomas vestibulares, donde el tratamiento profiláctico ha demostrado escaso beneficio en la prevención de las crisis de vértigo [63]. La terapia física, el estímulo $\mathrm{OKN}$ y la realidad virtual, han conseguido mejorar síntomas tan frecuentes en estos casos como la intolerancia al movimiento de cabeza y el vértigo visual $[64,65,66]$.

\section{CONCLUSIONES}

La RV es segura, efectiva y beneficiosa para los pacientes con mareo/desequilibrio, mejorando la sintomatología vertiginosa, el equilibrio, el control postural, el riesgo de caídas y restableciendo la calidad de vida $[10,56]$. Incluso, la RV influencia positivamente sobre el componente emocional que con frecuencia conlleva esta patología, disminuyendo la necesidad de tratamiento farmacológico o psicoterápico $[8,67]$. En definitiva, la RV representa actualmente el pilar fundamental de tratamiento de estos pacientes, aunque es posible que el conocimiento fisiopatológico y las nuevas tecnologías, permitan en el futuro ampliar el espectro y la especificidad de esta terapia $[9,68]$.

\section{AGRADECIMIENTOS}

Al Servicio de Rehabilitación del Hospital Clínico Universitario de Valladolid por el compromiso compartido de introducir la Rehabilitación Vestibular dentro del tratamiento fisioterápico general.

\section{BIBLIOGRAFÍA}

1. Shepard NT, Telian SA. Programmatic vestibular rehabilitation. Otolaryngol Head Neck Surg. 1995 Jan;112(1):173-82. 


\section{TERAPIA FÍSICA EN LA HIPOFUNCIÓN VESTIBULAR UNILATERAL Y BILATERAL BENITO-OREJAS J I ET AL}

2. Herdman SJ, Blatt P, Schubert MC, Tusa RJ. Falls in patients with vestibular deficits. Am J Otol. 2000 Nov;21(6):847-51.

3. Stevens JA, Corso PS, Finkelstein EA, Miller TR. The costs of fatal and non-fatal falls among older adults. Inj Prev. 2006 Oct;12(5):290-5.

4. Han BI, Song HS, Kim JS. Vestibular rehabilitation therapy: review of indications, mechanisms, and key exercises. J Clin Neurol. 2011 Dec;7(4):184-96.

5. Cawthorne T. Vestibular Injuries. Proc R Soc Med. 1946 Mar;39(5):270-3.

6. Cooksey FS. Rehabilitation in Vestibular Injuries. Proc R Soc Med. 1946 Mar;39(5):273-8.

7. Whitney SL, Alghwiri A, Alghadir A. Physical therapy for persons with vestibular disorders. Curr Opin Neurol. 2015 Feb;28(1):61-8.

8. Hall CD, Herdman SJ, Whitney SL, Cass SP, Clendaniel RA, Fife TD, et al. Vestibular Rehabilitation for Peripheral Vestibular Hypofunction: An Evidence-Based Clinical Practice Guideline: FROM THE AMERICAN PHYSICAL THERAPY ASSOCIATION NEUROLOGY SECTION. J Neurol Phys Ther. 2016 Apr;40(2):124-55.

9. Sulway S, Whitney SL. Advances in Vestibular Rehabilitation. Adv Otorhinolaryngol. 2019;82:164-169.

10. McDonnell MN, Hillier SL. Vestibular rehabilitation for unilateral peripheral vestibular dysfunction. Cochrane Database Syst Rev. 2015 Jan 13;1:CD005397.

11. Benito-Orejas J, Poncela-Blanco M, Diez-González L, Álvarez-Otero R, Aguilera-Aguilera G, Intraprendente-Martini J, Racines-Alava E, Martínez R, Marco-Carmona M, Sánchez-Gómez H, Yáñez-González R, González-Aguado R, Franco-Gutiérrez V, Guerra-Jiménez G, MazónGutierrez A, Gancedo S, Pérez R, Santos-Gorjón P, Sánchez-Blanco C, García-Purriños F, GascónRubio C, Salazar F, Bauer M, Valda-Rodrigo J, Mena-Domínguez E, Trinidad-Ruiz G, BatuecasCaletrío A. Guía práctica del vértigo posicional paroxístico. Revista ORL [Internet]. 1 Sep 2017 [citado 25 Abr 2019]; 8(3): 157-196. Disponible en: http://revistas.usal.es/index.php/2444-7986/ article/view/15655.
12. Cullen KE. The vestibular system: multimodal integration and encoding of self-motion for motor control. Trends Neurosci. 2012 Mar;35(3):185-96.

13. Eleftheriadou A, Skalidi N, Velegrakis GA. Vestibular rehabilitation strategies and factors that affect the outcome. Eur Arch Otorhinolaryngol. 2012 Nov;269(11):2309-16.

14. Alghadir AH, Iqbal ZA, Whitney SL. An update on vestibular physical therapy. J Chin Med Assoc. 2013 Jan;76(1):1-8.

15. Paige GD. Senescence of human visual-vestibular interactions: smooth pursuit, optokinetic, and vestibular control of eye movements with aging. Exp Brain Res. 1994;98(2):355-72.

16. Schubert MC, Migliaccio AA, Clendaniel RA, Allak A, Carey JP. Mechanism of dynamic visual acuity recovery with vestibular rehabilitation. Arch Phys Med Rehabil. 2008 Mar;89(3):500-7.

17. Viirre E, Sitarz R. Vestibular rehabilitation using visual displays: preliminary study. Laryngoscope. 2002 Mar;112(3):500-3.

18. Herdman SJ. Exercise strategies for vestibular disorders. Ear Nose Throat J. 1989 Dec;68(12):961-4.

19. Whitney SL, Sparto PJ, Hodges LF, Babu SV, Furman JM, Redfern MS. Responses to a virtual reality grocery store in persons with and without vestibular dysfunction. Cyberpsychol Behav. 2006 Apr;9(2):152-6.

20. Kramer PD, Roberts DC, Shelhamer M, Zee DS. A versatile stereoscopic visual display system for vestibular and oculomotor research. J Vestib Res. 1998 Sep-Oct;8(5):363-79.

21. Herdman SJ. Role of vestibular adaptation in vestibular rehabilitation. Otolaryngol Head Neck Surg. 1998 Jul;119(1):49-54.

22. Fetter M, Zee DS, Proctor LR. Effect of lack of vision and of occipital lobectomy upon recovery from unilateral labyrinthectomy in rhesus monkey. J Neurophysiol. 1988 Feb;59(2):394-407.

23. Herdman SJ, Schubert MC, Tusa RJ. Role of central preprogramming in dynamic visual acuity with vestibular loss. Arch Otolaryngol Head Neck Surg. 2001 Oct;127(10):1205-10. 


\section{TERAPIA FÍSICA EN LA HIPOFUNCIÓN VESTIBULAR UNILATERAL Y BILATERAL BENITO-OREJAS J I ET AL}

24. Schubert MC, Hall CD, Das V, Tusa RJ, Herdman SJ. Oculomotor strategies and their effect on reducing gaze position error. Otol Neurotol. 2010 Feb;31(2):228-31.

25. Herdman SJ. Advances in the treatment of vestibular disorders. Phys Ther. 1997 Jun;77(6):602-18.

26. Lisberger SG, Miles FA, Optican LM. Frequencyselective adaptation: evidence for channels in the vestibulo-ocular reflex? J Neurosci. 1983 Jun;3(6):1234-44.

27. Schubert MC, Zee DS. Saccade and vestibular ocular motor adaptation. Restor Neurol Neurosci. 2010;28(1):9-18.

28. Barnes GR. Visual-vestibular interaction in the control of head and eye movement: the role of visual feedback and predictive mechanisms. Prog Neurobiol. 1993 Oct;41(4):435-72.

29. Herdman SJ, Hall CD, Schubert MC, Das VE, Tusa RJ. Recovery of dynamic visual acuity in bilateral vestibular hypofunction. Arch Otolaryngol Head Neck Surg. 2007 Apr;133(4):383-9.

30. Macdougall HG, Curthoys IS. Plasticity during Vestibular Compensation: The Role of Saccades. Front Neurol. 2012 Feb 28;3:21.

31. Bartolomeo M, Biboulet R, Pierre G, Mondain M, Uziel A, Venail F. Value of the video head impulse test in assessing vestibular deficits following vestibular neuritis. Eur Arch Otorhinolaryngol. 2014 Apr;271(4):681-8.

32. Pfaltz CR. Vestibular compensation. Physiological and clinical aspects. Acta Otolaryngol. 1983 May-Jun;95(5-6):402-6.

33. Herdman S, Whitney S. Physical therapy treatment vestibular hypofunction. En: Herdman S, Clendaniel R. Vestibular Rehabilitation. $4^{\mathrm{a}} \mathrm{ed}$. Philadelphia: FA Davis Co;2014. p.394-431.

34. King L, Horak F. The role of vestibular system in postural control. En: Herdman S, Clendaniel R. Vestibular Rehabilitation. $4^{\text {a }}$ ed. Philadelphia: FA Davis Co;2014. p.29-48.

35. Horak FB, Nashner LM, Diener HC. Postural strategies associated with somatosensory and vestibular loss. Exp Brain Res. 1990;82(1):167-77.

36. Heusel-Gillig L, Hall C. Physical therapy management people with non-vestibular dizziness.
En: Herdman S, Clendaniel R. Vestibular Rehabilitation. $4^{\text {a }}$ ed. Philadelphia: FA Davis Co;2014. p.569-89.

37. Dozza M, Wall C 3rd, Peterka RJ, Chiari L, Horak FB. Effects of practicing tandem gait with and without vibrotactile biofeedback in subjects with unilateral vestibular loss. J Vestib Res. 2007;17(4):195-204.

38. Wall C 3rd, Wrisley DM, Statler KD. Vibrotactile tilt feedback improves dynamic gait index: a fall risk indicator in older adults. Gait Posture. 2009 Jul;30(1):16-21.

39. McGibbon CA, Krebs DE, Wolf SL, Wayne PM, Scarborough DM, Parker SW. Tai Chi and vestibular rehabilitation effects on gaze and wholebody stability. J Vestib Res. 2004;14(6):467-78.

40. Gabilan YP, Perracini MR, Munhoz MS, Gananç FF. Aquatic physiotherapy for vestibular rehabilitation in patients with unilateral vestibular hypofunction: exploratory prospective study. J Vestib Res. 2008;18(2-3):139-46.

41. Herdmman S, Clendaniel R. Physical therapy management of bilateral vestibular hypofunction and loss. En: Herdman S, Clendaniel R. Vestibular Rehabilitation. $4^{\mathrm{a}}$ ed. Philadelphia: FA Davis Co;2014. p.432-56.

42. Brandt T, Bronstein AM. Cervical vertigo. J Neurol Neurosurg Psychiatry. 2001 Jul;71(1):8-12.

43. Valda-Rodrigo J, Benito-Orejas JI, Alonso-VielbaVarea J. Revisión sobre el vértigo cervical. Revista ORL [Internet]. 18 Nov 2017 [citado 28 Abr 2019]; 9(2): 97-104. Disponible en: http://revistas. usal.es/index.php/2444-7986/article/view/17191.

44. Bronstein AM, Hood JD. The cervico-ocular reflex in normal subjects and patients with absent vestibular function. Brain Res. 1986 May 14;373(1-2):399-408.

45. Lundin-Olsson L, Nyberg L, Gustafson Y. "Stops walking when talking" as a predictor of falls in elderly people. Lancet. 1997 Mar 1;349(9052):617.

46. Keim RJ, Cook M, Martini D. Balance rehabilitation therapy. Laryngoscope. 1992 Nov;102(11):1302-7.

47. Goudakos JK, Markou KD, Psillas G, Vital V, Tsaligopoulos M. Corticosteroids and vestibular exercises in vestibular neuritis. Single-blind 


\section{TERAPIA FÍSICA EN LA HIPOFUNCIÓN VESTIBULAR UNILATERAL Y BILATERAL BENITO-OREJAS J I ET AL}

randomized clinical trial. JAMA Otolaryngol Head Neck Surg. 2014 May;140(5):434-40.

48. Hong SK, Kim JH, Kim HJ, Lee HJ. Changes in the gray matter volume during compensation after vestibular neuritis: a longitudinal VBM study. Restor Neurol Neurosci. 2014;32(5):663-73.

49. Deveze A, Bernard-Demanze L, Xavier F, Lavieille JP, Elziere M. Vestibular compensation and vestibular rehabilitation. Current concepts and new trends. Neurophysiol Clin. 2014 Jan;44(1):49-57.

50. Garcia AP, Ganança MM, Cusin FS, Tomaz A, Ganança FF, Caovilla HH. Vestibular rehabilitation with virtual reality in Ménière's disease. Braz J Otorhinolaryngol. 2013 May-Jun;79(3):366-74.

51. Meldrum D, Herdman S, Moloney R, Murray D, Duffy D, Malone K, et al. BMC Ear Nose Throat Disord. 2012 Mar 26;12:3. doi: 10.1186/14726815-12-3.

52. Sparrer I, Duong Dinh TA, Ilgner J, Westhofen M. Vestibular rehabilitation using the Nintendo ${ }^{\circledR}$ Wii Balance Board -- a user-friendly alternative for central nervous compensation. Acta Otolaryngol. 2013 Mar;133(3):239-45.

53. Verdecchia DH, Mendoza M, Sanguineti F, Binetti AC. Outcomes after vestibular rehabilitation and $\mathrm{Wii}^{\oplus}$ therapy in patients with chronic unilateral vestibular hypofunction. Acta Otorrinolaringol Esp. 2014 Nov-Dec;65(6):339-45.

54. Cohen AH. Vision rehabilitation for visual-vestibular dysfunction: the role of the neuro-optometrist. NeuroRehabilitation. 2013;32(3):483-92.

55. Pavlou M, Bronstein AM, Davies RA. Randomized trial of supervised versus unsupervised optokinetic exercise in persons with peripheral vestibular disorders. Neurorehabil Neural Repair. 2013 Mar-Apr;27(3):208-18.

56. Porciuncula F, Johnson CC, Glickman LB. The effect of vestibular rehabilitation on adults with bilateral vestibular hypofunction: a systematic review. J Vestib Res. 2012;22(5-6):283-98.

57. Phillips JO, Ling L, Nie K, Jameyson E, Phillips CM, Nowack AL, et al. Vestibular implantation and longitudinal electrical stimulation of the semicircular canal afferents in human subjects. J Neurophysiol. 2015 Jun 1;113(10):3866-92.
58. Sienko KH, Balkwill MD, Oddsson LI, Wall C 3rd. The effect of vibrotactile feedback on postural sway during locomotor activities. J Neuroeng Rehabil. 2013 Aug 9;10:93.

59. Dozza M, Horak FB, Chiari L. Auditory biofeedback substitutes for loss of sensory information in maintaining stance. Exp Brain Res. 2007 Mar;178(1):37-48.

60. Schneider KJ, Meeuwisse WH, Nettel-Aguirre A, Barlow K, Boyd L, Kang J, et al. Cervicovestibular rehabilitation in sport-related concussion: a randomised controlled trial. Br J Sports Med. 2014 Sep;48(17):1294-8.

61. Alsalaheen BA, Whitney SL, Mucha A, Morris LO, Furman JM, Sparto PJ. Exercise prescription patterns in patients treated with vestibular rehabilitation after concussion. Physiother Res Int. 2013 Jun;18(2):100-8.

62. Balci BD, Akdal G, Yaka E, Angin S. Vestibular rehabilitation in acute central vestibulopathy: a randomized controlled trial. J Vestib Res. 2013;23(4-5):259-67.

63. Radtke A, von Brevern $M$, Neuhauser $H$, Hottenrott T, Lempert T. Vestibular migraine: long-term follow-up of clinical symptoms and vestibulo-cochlear findings. Neurology. 2012 Oct 9;79(15):1607-14.

64. Bisdorff AR. Management of vestibular migraine. Ther Adv Neurol Disord. 2011 May;4(3):183-91.

65. Vitkovic J, Winoto A, Rance G, Dowell R, Paine M. Vestibular rehabilitation outcomes in patients with and without vestibular migraine. J Neurol. 2013 Dec;260(12):3039-48.

66. Pavlou M, Quinn C, Murray K, Spyridakou C, Faldon M, Bronstein AM. The effect of repeated visual motion stimuli on visual dependence and postural control in normal subjects. Gait Posture. 2011 Jan;33(1):113-8.

67. Meli A, Zimatore G, Badaracco C, De Angelis E, Tufarelli D. Effects of vestibular rehabilitation therapy on emotional aspects in chronic vestibular patients. J Psychosom Res. 2007 Aug;63(2):185-90.

68. Tjernström F, Zur O, Jahn K. Current concepts and future approaches to vestibular rehabilitation. J Neurol. 2016 Apr;263 Suppl 1:S65-70. 